\title{
EFFECT OF DIETARY Agaricus bisporus AND Auricularia auricula CRUDE EXTRACT SUPLEMENTATION ON CARCASS QUALITY OF BROILER
}

\author{
M. Halim Natsir*1), Osfar Sjofjan ${ }^{1)}$, Reynaldy H. Ardyansyah ${ }^{2}$ ) \\ 1) Lecturer in Animal Science Faculty, Brawijaya University, Jl. Veteran, Ketawanggede, Kec. Lowokwaru, \\ Malang City, East Java, Indonesia, 65145 \\ 2) Postgraduate student in Animal Science Faculty, Brawijaya University, Jl. Veteran, Ketawanggede, Kec. \\ Lowokwaru, Malang City, East Java, Indonesia, 65145 \\ Email: emhanatasir@ub.ac.id
}

Submitted 15 June 2020; Accepted 27 July 2020

\begin{abstract}
This research was conducted to examine Agaricus bisporus and Auricularia auricula crude extract using different solvents (water, ethanol, and methanol) on infrared spectroscopy absorbance during extraction and the impact on broiler carcass. Agaricus bisporus and Auricularia auricula crude extracts were scanned using fourier transform infrared (FT-IR) spectroscopy. Each mushroom crude extract was chosen and applied into broiler diets as feed additive at $0 \%, 0.4 \%, 0.8 \%, 1.2 \%$, and compared zinc bacitracin inclusion. Variable measured were final live weight, carcass yield, breast meat yield, and abdominal fat yield of broiler. Two hundred and forty day-old chicks were randomly allocated into eight dietary treatments, each treatment was replicated three times with ten chicks for each pen. Diets and water were offered ad libitum. Methanolic extract showed monosaccharide absorption peak in fingerprint region at wavelength $890 \mathrm{~cm}^{-1}, 930 \mathrm{~cm}^{-1}, 1050 \mathrm{~cm}^{-1}, 1150 \mathrm{~cm}^{-1}$ which indicates alpha and beta linkage than the others solvent. Even so, dietary inclusion of methanolic extracts of Agaricus bisporus and Auricularia auricula did not show any effect on final live weight and the yiled of carcass, breast meat and abdominal fat of broiler. In conclusion, methanolic extraction is effective to extract monosaccharides with $\alpha$ - and $\beta$ - linkages from Agaricus bisporus and Auricularia auricula, while the dietary inclusion of methanolic extracts of both edible mushroom and zinc bacitracin has no effect on carcass quality of broilerin broiler diets did not show differences between treatments as well as zinc bacitracin group.
\end{abstract}

Keyword: Antibiotics; broiler carcass; mushroom extract; prebiotics; phytobiotics 


\section{INTRODUCTION}

The use of antibiotic growth promoter (AGP) in poultry and cattle industry in Indonesia has been banned since early 2018 . Common AGP used in poultry industry was zinc bacitracin which is used to optimize broiler growth. Zinc bacitracin usage in long term in poultry diets may induce the deposition of the antibiotic residue to poultry products such as meat and egg. Antibiotics resdidue in poultry products may impact on human immune system against antimicrobial-resistance bacteria. There has been much research evaluating some alternative ways to replace AGP in poultry production. In this study, glucans from the mushroom play a role as prebiotic.

Agaricus bisporus and Auricularia auricula are two kinds of edible mushroom which contain many therapeutic polysaccharides. Most polysaccharide in Agaricus bisporus and Auricularia auricula are in the form of glucan. $\beta$-glucan from Agaricus bisporus and Auricularia auricula activate macrophages cell and may improve host immunity (Minato and Abe, 2013). Conventional extraction is usually performed in long time period of time (more than $2 \mathrm{~h}$ ) at reflux temperature $90^{\circ} \mathrm{C}$. While microwave assisted extraction may increase yield extract at short time and reduce solvent usage (Proestos and Komaitis, 2008). Water usage as solvent will absorb more microwave energy, therefore solvent polarity is very important to be considered. Common polar solvents showed better result in polysaccharides extraction by using microwave assisted extraction than nonpolar solvents (Wang and Weller, 2006).

Agaricus bisporus and Auricularia auricula are two kinds of mushroom which cultivate in subtropical climate such as
Indonesia. Liu et. al. (2013) reported that ethanolic extract of Agaricus bisporus showed better antoxidant activity against various radical scavengers, and Agaricus bisporus ethanolic extract could be natural antioxidant and therapeutic agents. Infrared spectra of Auricularia auricula extracted using microwave assisted extraction showed stretching vibration of $-\mathrm{OH}$ and $\mathrm{C}-\mathrm{H}$ at about $3600-3200 \mathrm{~cm}^{-1}$ and $300-2800 \mathrm{~cm}^{-1}$, then the absorption at $903 \mathrm{~cm}^{-1}$ indicates $\beta$ glycosidic linkages as $\beta$-glucan and the peak at 1145,1075 , and $1042 \mathrm{~cm}^{-1}$ showed sugar form as pyranose. Microwave assisted extraction could influence the molecular weight of the polysaccharide and the molecular weight changes could affect the bioactivity of the polysaccharide (Zeng et. al., 2012; Feng et. al., 2010). The combination between $\beta$-glucan and antibiotic addition into weanling pigs diets could increase dry matter, gross energy, crude protein, and crude fat digestibility (Hahn et. al., 2006).

Agaricus bisporus is known to play a role as immunomodulatory agent and growth performance of broiler and turkey (Guimarães et al., 2014; Giannenas et al., 2011). Agaricus bisporus additional in laying hen diets showed $2 \%$ residual stem from white button mushroom improve laying hens performance and increase nutrient utilization (Yang et. al., 2020). Another study based on Shamsi et. al. (2015) reported that the inclusion of $0.2 \%$ powdered Agaricus bisporus into broiler diets significantly reduced daily feed intake and feed conversion ratio. The lack study investigated influence of Agaricus bisporus and Auricularia auricula crude extract in broiler diets to replace AGP in broiler diets is required. The objective of this study was to investigate Agaricus bisporus and
*Corresponding author:

M. Halim Natsir

Email: emhanatasir@ub.ac.id

Lecturer in Animal Science Faculty, Brawijaya University, Jl. Veteran, Ketawanggede, Kec. Lowokwaru, Malang City, East Java, Indonesia, 65145
How to cite:

Natsir, M. H., Sjofjan, O., Ardyansah, R. H. (2020). Effect of Dietary Agaricus bisporus and Auricularia auricula Crude Extract Suplementation on Carcass Quality of Broiler. Jurnal Ilmu dan Teknologi Hasil Ternak, 15 (3), 183-193 
Auricularia auricula crude extract structure changes during extraction process using microwave assisted extraction (MAE) with different solvents by infrared spectroscopy and the impact of the inclusion of the mushroom extracts in diets on the carcass quality of broiler.

\section{MATERIALS AND METHODS}

\section{Mushroom preparation and extraction}

Agaricus bisporus and Auricularia auricula were purchased from local mushroom farmer in Singosari sub district, Malang District, East Java, Indonesia. The fresh mushroom were sliced to reduce the size, then soaked using three different solvents (water, ethanol 96\%, and methanol $70 \%$ ) for $24 \mathrm{~h}$. Extraction was performed using a modified microwave $(50 \mathrm{~Hz}$, 399 watt) (Purwanto et. al., 2010) with additional equipment vacum pump. MAE of the edible mushroom was done at $50^{\circ} \mathrm{C}$ for
15 min. The crude extract was then filtered using cheese cloth and its extraction schematics is shown in Fig. 1. The filtrate was evaporated using MAE for second time for $15 \mathrm{~min}$ to get crude extract from $A$. bisporus and $A$. auricula. Crude mushroom extract was stored into freezer at $-20^{\circ} \mathrm{C}$ for in-vivo trial. Both mushroom crude extracts were centrifuged under $3500 \mathrm{rpm}$ at about $15 \mathrm{~min}$, then separate between supernatant and solid fraction. Solid fraction after centrifuged were dried using microwave under $50^{\circ} \mathrm{C}$ for $10 \mathrm{~min}$. Then $3 \mathrm{mg}$ of powdered Agaricus bisporus and Auricularia auricula crude extract mix with $97 \mathrm{mg}$ of $\mathrm{KBr}$ (Merck KGaA, 54271 Darmstadt, Germany) then pressed by using $\mathrm{KBr}$ press holder. Finally the spectra was scanned in wavelength range 500-4000 $\mathrm{cm}^{-1}$ with FT-IR spectrofotometer. FT-IR analysis was performed at central laboratory of State University of Malang (Experiment 1).

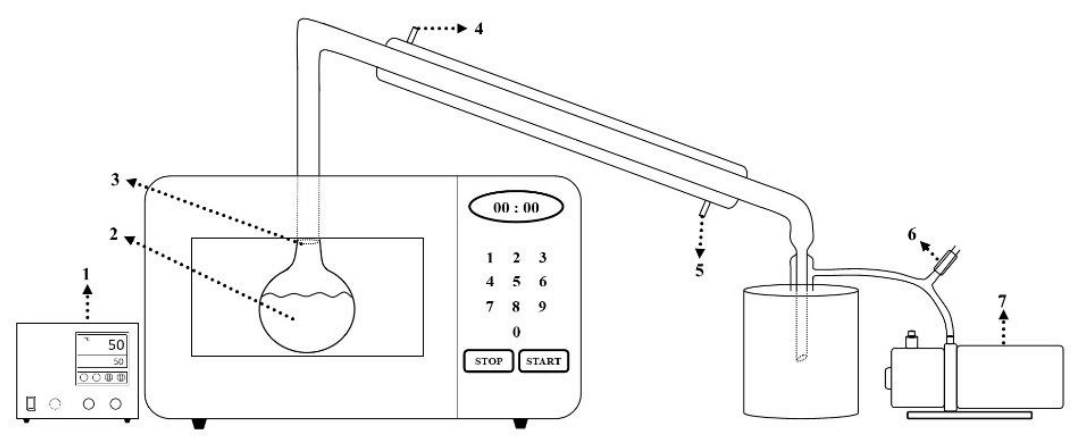

Figure 1. Microwave assisted extraction schematics (1: thermo control; 2: solvent and symplisia; 3: removeable flask; 4: cooling water outlet; 5: cooling water inlet; 6 : air controller; 7: vacuum pump)

\section{Animals and experimental design}

Two hundred and forty day-old chicks (unsex) were randomly allocated into 8 experimental groups with average body weight of $37,6 \pm 2,90 \mathrm{~g} / \mathrm{chick}$. Three replication were done for each treatment with ten chicks in each pen. The dimensions of the pen were $1 \times 1,2 \times 0,8 \mathrm{~m}^{3}$ and equipped by hanging feeder and drinker. The feed and water given ad libitum. During ten days, all chicks got brooding phase with controlled room temperature at $26-35^{\circ} \mathrm{C}$ and humidity $65 \%-75 \%$. The experiment was done in $35 \mathrm{~d}$. Basal diet BR1 was given at the first $21 \mathrm{~d}$ (starter period), and BR2 was given later until $35 \mathrm{~d}$ (finisher period), basal diets purcashed from PT. Japfa Comfeed, Indonesia. Chemical composition of the basal diet is shown in Table 1.

The 8 experimental group were control (P0), basal diets containing zinc bacitracin as positive control (P1), basal diets containing Agaricus bisporus crude extract of $0.4 \%(\mathrm{P} 2), 0.8 \%(\mathrm{P} 3), 1.2 \%$ (P4), and basal diets containing Auricularia auricula crude extract of $0.4 \%$ (T5), 
$0.8 \%$ (T6), $1.2 \%$ (T7). Each experimental feed were prepared daily and given twice a day in the morning and afternoon, then mushroom crude extract was sprayed onto the basal diets (Experiment 2).

Table 1. Chemical composition of basal diets (BR1 and BR2) .

\begin{tabular}{lcc}
\hline Chemical composition & BR1 $^{\mathrm{c}}$ & BR2 $^{\mathrm{d}}$ \\
\hline Dry matter $(\%)$ & 86.58 & 86.49 \\
Crude Protein $(\%)^{\mathrm{b}}$ & 23.09 & 20.93 \\
${\text { Crude fat }(\%)^{\mathrm{b}}}_{\left.\text {Crude fiber }^{\mathrm{b}} \%\right)^{\mathrm{b}}}$ & 6.07 & 5.25 \\
Ash $(\%)^{\mathrm{b}}$ & 3.55 & 5.25 \\
\hline
\end{tabular}

${ }^{a}$ Based on Animal Feed Laboratory, Faculty of Animal Science, Universitas Brawijaya

${ }^{\mathrm{b}}$ Based on $100 \%$ dry matter

${ }^{\mathrm{c}}$ Ingredients: Corn, SBM, MBM, CGM, Palm olein, Lysine, Methionine sodium bicarbonate, Vitamin, Calcium, Phosphorus, Mineral.

${ }^{\mathrm{d}}$ Ingredients: Corn, SBM, MBM, CGM, CPO, Essential amino acid, Essential mineral, Premix, Vitamin.

\section{Broiler carcass measurements}

The spectra of Agaricus bisporus and Auricularia auricula crude extract using FTIR spectroscopy to investigate the absorption banding from polysaccharides of both edible mushroom crude extracts. Meanwhile, in experiment 2 on day 35, the broilers were weighed and sampling was performed by marking a broiler with average weight for every pen. The samples were then slaughtered. The yield of the carcass, breast meat and abdominal fat was determined according to the following formula:

Carcass $(\%)=\frac{\text { Carcass weight }(\mathrm{g})}{\text { Live weight }(\mathrm{g})} \times 100$

Breast meat (\%)

$$
\begin{aligned}
& =\frac{\text { Filleted breast meat }(\mathrm{g})}{\text { Carcass weight }(\mathrm{g})} \\
& \times 100
\end{aligned}
$$

abdominal fat (\%)

$$
=\frac{\text { Abdominal fat }(\mathrm{g})}{\text { Live weight }(\mathrm{g})} \times 100
$$

\section{Statistical analysis}

The spectrum data from FT-IR spectroscopy analysis (experiment 1) were analyzed descriptively. Meanwhile, the data from experiment 2 were analyzed using a one-way ANOVA with a completely randomized design (CRD). The orthogonal contrast tests were performed later for all variables using Minitab ${ }^{\circledR} 18.1$ (Minitab Inc, USA).

\section{RESULTS AND DISCUSSION}

Infrared spectrum of Agaricus bisporus crude extract are shown in Fig. 2 and Table 2. Agaricus bisporus crude extract showed peak at $930 \mathrm{~cm}^{-1}$ stretching vibration which indicates the presence of $\beta$-1,4-glucan. Then peak at $850 \mathrm{~cm}^{-1}$ in $\mathrm{KAA}, \mathrm{KAE}$, and KAM which is indicates the existence of alpha bound linkages in fingerprint region. $\mathrm{KAE}$ showed strongest alpha banding of monosaccharides that is alpha peak absorption at $893,0 \mathrm{~cm}^{-1}$ while KAA and KAM alpha peak absorption at 848,7 and $852,5 \mathrm{~cm}^{-1}$. It indicates that solvent usage may dilute $\alpha$-glucan from Agaricus bisporus. El-Batal et. al. (2008) stated that absorption on fingerprint region at $890 \mathrm{~cm}^{-1}$ indicates $1,3-\beta$-glucan banding, while absorption at $930 \mathrm{~cm}^{-1}$ indicates $\beta(1,4)$ polysaccharide.

The band attributed to $\mathrm{C}-\mathrm{O}$ stretching vibration appeared at wavelength $1045 \mathrm{~cm}^{-}$ ${ }^{1}$, where is KAE did not show any absorption in these wavelength region. The group of $\mathrm{C}$ $\mathrm{H}$ banding appeared as absorption peaked at $1085,9 \mathrm{~cm}^{-1}$ in KAC and $1099,4 \mathrm{~cm}^{-1}$ in 
KAM, which KAA and KAE did not show $\mathrm{C}-\mathrm{H}$ group in these wavelength. Stretching vibration at wavelength $1150 \mathrm{~cm}^{-1}$ showed $\mathrm{C}-\mathrm{O}-\mathrm{C}$ banding group, stretching vibration of $\mathrm{C}-\mathrm{O}-\mathrm{C}$ in KAC appeared at wavelength $1147,7 \mathrm{~cm}^{-1}$, KAA at wavelength $1122,6 \mathrm{~cm}^{-1}$, KAE at $1149,6 \mathrm{~cm}^{-1}$, while KAM showed stronger C-O-C stretching vibration banding group at wavelength $1166,9 \mathrm{~cm}^{-1}$. Wavelength range at 1175 $1140 \mathrm{~cm}^{-1}$ indicate that $\mathrm{C}-\mathrm{O}$ banding group appeared at $1147 \mathrm{~cm}^{-1}$ with medium intensity as $\alpha$-D-glucose, and appear absorption at $1153 \mathrm{~cm}^{-1} \mathrm{C}-\mathrm{O}$ banding group as $\beta$-D-glucose that is monosaccharide molecule (Nikonenko et. al., 2000).

Table 2. FT-IR spectra of $A$. bisporus crude extract

\begin{tabular}{|c|c|c|c|c|c|}
\hline \multicolumn{3}{|c|}{ A. bisporus (KAC) } & \multicolumn{3}{|c|}{ A. bisporus crude water extract (KAA) } \\
\hline $\begin{array}{l}\text { Wavelength } \\
\left(\mathrm{cm}^{-1}\right)\end{array}$ & Group & $\begin{array}{c}\text { Type of } \\
\text { Vibration }\end{array}$ & $\begin{array}{l}\text { Wavelength } \\
\left(\mathrm{cm}^{-1}\right)\end{array}$ & Group & $\begin{array}{c}\text { Type of } \\
\text { Vibration }\end{array}$ \\
\hline & & & 848,7 & alpha chain & \\
\hline 931.6 & beta 1.4 glucan & & 929.7 & beta 1.4 glucan & \\
\hline 1026.1 & $\mathrm{C}-\mathrm{O}$ & stretching & 1043.5 & $\mathrm{C}-\mathrm{O}$ & stretching \\
\hline 1085.9 & $\mathrm{C}-\mathrm{H}$ & bending & & & \\
\hline 1147.7 & $\mathrm{C}-\mathrm{O}-\mathrm{C}$ & stretching & 1122.6 & $\mathrm{C}-\mathrm{O}-\mathrm{C}$ & stretching \\
\hline \multirow[t]{2}{*}{2873.9} & $\mathrm{C}-\mathrm{H}$ & stretching & 2875.9 & $\mathrm{C}-\mathrm{H}$ & stretching \\
\hline & & & 3263.6 & $\mathrm{O}-\mathrm{H}$ & stretching \\
\hline \multicolumn{3}{|c|}{ A. bisporus crude ethanol extract (KAE) } & \multicolumn{3}{|c|}{ A. bisporus crude methanol extract (KAM) } \\
\hline $\begin{array}{c}\text { Wavelength } \\
\left(\mathrm{cm}^{-1}\right)\end{array}$ & Group & $\begin{array}{c}\text { Type of } \\
\text { Vibration }\end{array}$ & $\begin{array}{l}\text { Wavelength } \\
\left(\mathrm{cm}^{-1}\right)\end{array}$ & Group & $\begin{array}{c}\text { Type of } \\
\text { Vibration }\end{array}$ \\
\hline 893.0 & alpha chain & & 852.5 & alpha chain & \\
\hline \multirow[t]{3}{*}{935.5} & beta 1.4 glucan & & 937.4 & beta 1.4 glucan & \\
\hline & & & 1031.9 & $\mathrm{C}-\mathrm{O}$ & stretching \\
\hline & & & 1099.4 & $\mathrm{C}-\mathrm{H}$ & tekukan \\
\hline 1149.6 & $\mathrm{C}-\mathrm{O}-\mathrm{C}$ & stretching & 1166.9 & $\mathrm{C}-\mathrm{O}-\mathrm{C}$ & stretching \\
\hline 1429.3 & $\mathrm{C}-\mathrm{H}_{4}$ & bending & 1425.4 & $\mathrm{C}-\mathrm{H}_{4}$ & bending \\
\hline 1685.8 & $\mathrm{C}=\mathrm{O}$ & stretching & 1691.6 & $\mathrm{C}=\mathrm{O}$ & stretching \\
\hline 2873.9 & $\mathrm{C}-\mathrm{H}$ & stretching & 2953.0 & $\mathrm{C}-\mathrm{H}$ & stretching \\
\hline 3286.7 & $\mathrm{O}-\mathrm{H}$ & stretching & 3421.7 & $\mathrm{O}-\mathrm{H}$ & stretching \\
\hline
\end{tabular}

There are peak absorption of bending vibration $\mathrm{C}-\mathrm{H}$ alkana banding group in $\mathrm{KAE}$ $\left(1429,3 \mathrm{~cm}^{-1}\right)$ and $\operatorname{KAM}\left(1425,4 \mathrm{~cm}^{-1}\right)$, while KAC and KAA did not shown bending vibration of $\mathrm{C}-\mathrm{H}$ alkana banding group. KAE and KAM absorption peak appeared at wavelength $1685,8 \mathrm{~cm}^{-1}$ and $1691,6 \mathrm{~cm}^{-1}$ of $\mathrm{C}=\mathrm{O}$ stretching vibration banding group from monosaccharide. Stretching vibration of $\mathrm{C}-\mathrm{H}$ banding group appeared at wavelength 2873,9 cm-1 (KAC), 2875,9 cm-1 (KAA), 2873,9 (KAE), dan 2953,0 cm-1 (KAM), which is KAM showed stronger stretching vibration of $\mathrm{C}-\mathrm{H}$ banding group. Hydrogen bond of $-\mathrm{OH}$ stretching vibration group appeared at wavelength $3263,6 \mathrm{~cm}^{-1}$ on KAA, $3286,7 \mathrm{~cm}^{-1}$ on KAE, and $3421,7 \mathrm{~cm}^{-1}$ on KAM which is KAC did not show stretching vibration of -OH banding group. There was an absorption enlargement at $3000-3500 \mathrm{~cm}^{-}$ 1 for A. bisporus extract. Kozarski et. al. (2011) reported that polysaccharide from hot-water extraction of A. bisporus appear peak absorption at $3416,5 \mathrm{~cm}^{-1}$ indicate $-\mathrm{OH}$ group, then -CO group appear peak absorption at $1023 \mathrm{~cm}^{-1}$, and C-O-C banding group appeared at $1155 \mathrm{~cm}^{-1}$, and absorption 
at $850 \mathrm{~cm}^{-1}$ indicating $\alpha$ - banding group. There are different result between Agaricus species differences, Whereas Agaricus bisporus contain glucose compound more than $85 \%$ while agaricus brasiliensis contain glucose $58 \%$ and complex glycan. Which is $\alpha$-glucan in Agaricus brasiliensis higher than $\beta$-glucan (Kozarski et. al., 2011).

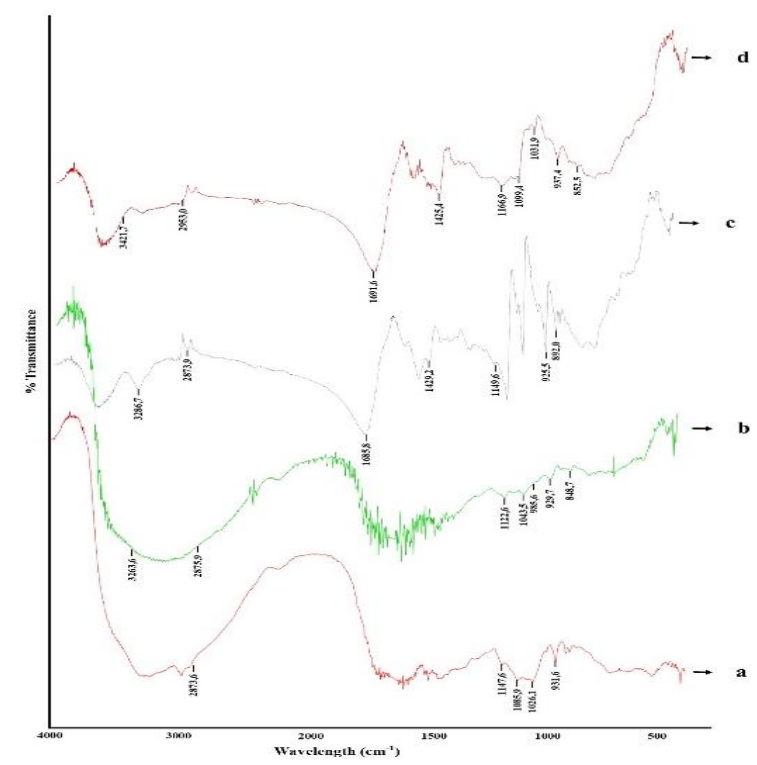

Figure 2. FT-IR spectroscopy of Agaricus bisporus crude extract (a: control group; b: water solvent; c: ethanol solvent; d: methanol solvent)

Table 3. FT-IR spectra of $A$. auricula crude extract

\begin{tabular}{|c|c|c|c|c|c|}
\hline \multicolumn{3}{|c|}{ A. auricula (KUC) } & \multicolumn{3}{|c|}{ A. auricula crude water extract (KUA) } \\
\hline $\begin{array}{l}\text { Wavelength } \\
\left(\mathrm{cm}^{-1}\right)\end{array}$ & Group & $\begin{array}{c}\text { Type of } \\
\text { Vibration }\end{array}$ & $\begin{array}{l}\text { Wavelength } \\
\left(\mathrm{cm}^{-1}\right)\end{array}$ & Group & $\begin{array}{c}\text { Type of } \\
\text { Vibration }\end{array}$ \\
\hline 895.0 & 1.3 beta glucan & & & & \\
\hline 1049.3 & $\mathrm{C}-\mathrm{O}$ & stretching & 1045.4 & $\mathrm{C}-\mathrm{O}$ & stretching \\
\hline 1082.1 & $\mathrm{C}-\mathrm{H}$ & bending & & & \\
\hline 1157.3 & $\mathrm{C}-\mathrm{O}-\mathrm{C}$ & stretching & & & \\
\hline 1253.7 & $\mathrm{C}-\mathrm{O}-\mathrm{C}$ & stretching & 1246.0 & $\mathrm{C}-\mathrm{O}-\mathrm{C}$ & stretching \\
\hline 1317.4 & $\mathrm{C}-\mathrm{H}$ & bending & & & \\
\hline 2895.2 & $\mathrm{C}-\mathrm{H}$ & stretching & 2852.7 & $\mathrm{C}-\mathrm{H}$ & stretching \\
\hline 3226.9 & $\mathrm{O}-\mathrm{H}$ & stretching & 3321.4 & $\mathrm{O}-\mathrm{H}$ & stretching \\
\hline \multicolumn{3}{|c|}{ A. auricula crude ethanol extract (KUE) } & \multicolumn{3}{|c|}{ A. auricula crude methanol extract (KUM) } \\
\hline $\begin{array}{l}\text { Wavelength } \\
\left(\mathrm{cm}^{-1}\right)\end{array}$ & Group & $\begin{array}{c}\text { Type of } \\
\text { Vibration }\end{array}$ & $\begin{array}{c}\text { Wavelength } \\
\left(\mathrm{cm}^{-1}\right)\end{array}$ & Group & $\begin{array}{c}\text { Type of } \\
\text { Vibration }\end{array}$ \\
\hline 935.5 & beta 1.4 glucan & & 929.7 & beta 1.4 glucan & \\
\hline 1041.6 & $\mathrm{C}-\mathrm{O}$ & stretching & 1041.6 & $\mathrm{C}-\mathrm{O}$ & stretching \\
\hline & & & 1076.3 & $\mathrm{C}-\mathrm{H}$ & bending \\
\hline 1145.7 & $\mathrm{C}-\mathrm{O}-\mathrm{C}$ & stretching & 1147.7 & $\mathrm{C}-\mathrm{O}-\mathrm{C}$ & stretching \\
\hline 1242.2 & $\mathrm{C}-\mathrm{O}-\mathrm{C}$ & stretching & 1242.2 & $\mathrm{C}-\mathrm{O}-\mathrm{C}$ & stretching \\
\hline 1317.4 & $\mathrm{C}-\mathrm{H}$ & bending & 1317.4 & $\mathrm{C}-\mathrm{H}$ & bending \\
\hline & & & 1745.6 & $\mathrm{C}=\mathrm{O}$ & stretching \\
\hline 2850.8 & $\mathrm{C}-\mathrm{H}$ & stretching & 2852.7 & $\mathrm{C}-\mathrm{H}$ & stretching \\
\hline
\end{tabular}


Infrared spectrum of Auricularia auricula crude extract are shown in Fig. 3 and Table 3. Auricularia auricula crude extract did not show peak at $890 \mathrm{~cm}^{-1}$ indicating the absence of $\beta$-glycosidic linkages. Meanwhile, KUC shown peak absorption at wavelength $895,0 \mathrm{~cm}^{-1}$ from $1,3-\beta$-glucan. However, the extract of $A$. auricula did not show absorption at these wavelengths as 1,3- $\beta$-glucan could not be diluted. KUE and KUM showed peak absorption at $935,48 \mathrm{~cm}^{-1}$ and $929,69 \mathrm{~cm}^{-1}$ which indicates $1,4-\beta$-glucan. 1,4- $\beta$-glucan in KUM had lowering peak absorption intensity, while KUC and KUA did not show peak absorption at these wavelength.

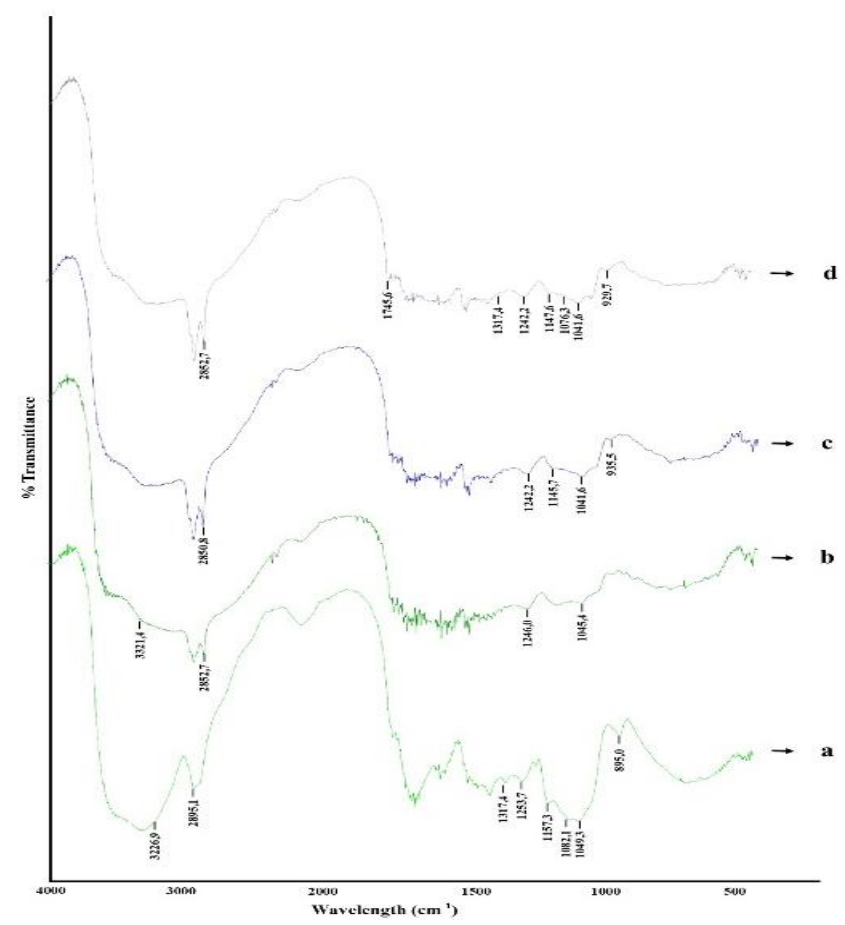

Figure 3. FT-IR spectrum of Auricularia auricula crude extract (a: control group; b: water solvent; c: ethanol solvent; d: methanol solvent)

Table 4. The effect of Agaricus bisporus and Auricularia auricula crude extract on broiler performance at 35 days of age ${ }^{\mathrm{a}}$.

\begin{tabular}{lcccc}
\hline Treatments & $\begin{array}{c}\text { Final live } \\
\text { weight (g/chick) }\end{array}$ & $\begin{array}{c}\text { Carcass yield } \\
(\%)\end{array}$ & $\begin{array}{c}\text { Breast meat } \\
\text { yield }^{\mathrm{b}}(\%)\end{array}$ & $\begin{array}{c}\text { Abdominal fat } \\
\text { yield }^{\mathrm{c}}(\%)\end{array}$ \\
\hline P0 & $2171 \pm 45.7$ & $73.5 \pm 1.8$ & $30.5 \pm 3.2$ & $1.41 \pm 0.38$ \\
P1 & $2163 \pm 3.0$ & $73.0 \pm 2.1$ & $31.0 \pm 3.6$ & $1.65 \pm 0.14$ \\
P2 & $2154 \pm 46.8$ & $72.9 \pm 0.6$ & $29.2 \pm 2.2$ & $1.20 \pm 0.13$ \\
P3 & $2217 \pm 39.5$ & $70.6 \pm 4.1$ & $29.1 \pm 3.0$ & $1.49 \pm 0.26$ \\
P4 & $2107 \pm 26.8$ & $71.9 \pm 2.4$ & $29.1 \pm 1.7$ & $1.19 \pm 0.09$ \\
P5 & $2163 \pm 54.8$ & $73.5 \pm 0.3$ & $29.9 \pm 0.7$ & $1.51 \pm 0.83$ \\
P6 & $2182 \pm 131.9$ & $72.4 \pm 3.0$ & $29.4 \pm 1.6$ & $1.72 \pm 0.16$ \\
P7 & $2138 \pm 90.7$ & $73.5 \pm 3.6$ & $30.5 \pm 1.9$ & $1.49 \pm 0.58$ \\
\hline
\end{tabular}

${ }^{\mathrm{a}}$ Results are given as means \pm standard deviation

${ }^{\mathrm{b}}$ Data are shown based on $100 \%$ carcass weight

${ }^{\mathrm{c}}$ Data are shown based on $100 \%$ live weight 
Table 5. Significance levels of orthogonal contrast tests on broiler's final weight and carcass quality ${ }^{a}$.

\begin{tabular}{lcccc}
\hline Treatments & $\begin{array}{c}\text { Live weight } \\
(\mathrm{g} / \text { chick })\end{array}$ & Carcass $(\%)$ & Breast meat $(\%)$ & $\begin{array}{c}\text { Abdominal fat } \\
(\%)\end{array}$ \\
\hline P0vsP1-P7 & 0.676 & 0.846 & 0.954 & 0.704 \\
P1vsP2-P7 & 0.797 & 0.567 & 0.608 & 0.837 \\
P2-P4vsP5-P7 & 0.939 & 0.733 & 0.346 & 0.395 \\
P2vsP3-P4 & 0.952 & 0.278 & 0.489 & 0.157 \\
P3vsP4 & 0.869 & 0.359 & 0.947 & 0.638 \\
P5vsP6-P7 & 0.055 & 0.553 & 0.969 & 0.377 \\
P6vsP7 & 0.944 & 0.751 & 0.989 & 0.732 \\
\hline
\end{tabular}

${ }^{a} P$-value on orthogonal contrast tests

Then peak absorption stretching vibration of C-O carbonyl group appeared at wavelength 1049,3 $\mathrm{cm}^{-1} \quad$ (KUC), $1045,0 \mathrm{~cm}^{-1}$ (KUA), 1041,6 $\mathrm{cm}^{-1}$ (KUE), and 1041,6 $\mathrm{cm}^{-1} \quad$ (KUM) (commonly appeared at wavelength $\left.1050-1300 \mathrm{~cm}^{-1}\right)$. The band attributed to bending vibration of C-H group appeared at wavelength 1082,1 and $1076,3 \mathrm{~cm}^{-1}$. The band attributed to C$\mathrm{O}-\mathrm{C}$ stretching vibration appeared at wavelength $1157,4 \mathrm{~cm}^{-1}$ in KUC, KUE and KUM showed reducing absorption intensity at wavelength 1145,7 dan $1147,7 \mathrm{~cm}^{-1}$. Meanwhile KAA did not show absorption peak at $1150 \mathrm{~cm}^{-1}$. Then appeared stretching vibration of $\mathrm{C}-\mathrm{O}-\mathrm{C}$ banding group at wavelength $1147,7 \mathrm{~cm}^{-1}$ in KAC, $1122,6 \mathrm{~cm}^{-1}$ in KAA, $1149,6 \mathrm{~cm}^{-1}$ in KAE, and stronger C-O-C banding group of KAM appeared at wavelength $1166,9 \mathrm{~cm}^{-1}$. The band attributed to bending vibration of $\mathrm{C}-\mathrm{H}$ alkana banding group appeared at wavelength $1429,3 \mathrm{~cm}^{-1}$ in KAE and $1425,4 \mathrm{~cm}^{-1}$ in KAM, while KAC and KAA did not show bending vibration of $\mathrm{C}-\mathrm{H}$ alkana banding group at these region. KAE and KAM showed peak absorption at wavelength 1685,8 dan $1691,6 \mathrm{~cm}^{-1}$ which is indicate of $\mathrm{C}=\mathrm{O}$ banding group. Then peak absorption appeared at wavelength $2873,9 \mathrm{~cm}^{-1}$ (KAC), 2875,9 $\mathrm{cm}^{-1}$ (KAA), $2873,9 \mathrm{~cm}^{-1}$ (KAE), and 2953,0 $\mathrm{cm}^{-1}$ (KAM) which indicates stretching vibration of $\mathrm{C}-\mathrm{H}$ banding group. Peak absorption at wavelength $3263,6 \mathrm{~cm}^{-1}$ pada KAA, $3286,7 \mathrm{~cm}^{-1}$ pada $\mathrm{KAE}$, and $3421,7 \mathrm{~cm}^{-1}$ in KAM showed stretching vibration hydrogen bond of -OH banding group. Which in KAC did not found stretching vibration of $-\mathrm{OH}$ banding group. Zeng et. al. (2012) reported that microwave assisted extraction technology on Auricularia auricula showed absorption peak at $3435 \mathrm{~cm}^{-1}$ and $2927 \mathrm{~cm}^{-1}$ that is indicate $-\mathrm{OH}$ stretching vibration and $\mathrm{C}-\mathrm{H}$ bending vibration. Then absorption peak appeared at $903 \mathrm{~cm}^{-1}$ represents $\beta$ glycosidic bond linkage. Based on Agaricus bisporus crude extract IR spectrum, methanolic extract showed absorption peak at $890 \mathrm{~cm}^{-1}$ representing 1,3- $\beta$-glycosidic linkages, $\quad 930 \quad \mathrm{~cm}^{-1} \quad$ representing $1,4-\beta$-glycosidic linkages, and $1150 \mathrm{~cm}^{-1}$ representing $\mathrm{C}-\mathrm{O}-\mathrm{C}$ stretching vibration, and the absorption peaks appeared in these regions indicate sugar conformation (Nikonenko et. al., 2000). Auricularia auricula water extract did not show absorption peak at $1150 \mathrm{~cm}^{-1}$ and $930 \mathrm{~cm}^{-1}$, while methanolic extract showed absorption peak at $1150 \mathrm{~cm}^{-1}$ and $930 \mathrm{~cm}^{-1}$.

Based on analysis of variance (ANOVA) there are no significant differences between treatments on carcass quality of broiler. The non significant differences caused of Agaricus bisporus and Auricularia auricula crude extract addition of $1.2 \%$ in broiler diets did not changes the nutrient utilization. In previous study Kavyani et. al. (2012) reported that $1 \%$ dry Agaricus bisporus inclusion in broiler diets significant increase broiler body weight at 21 days of rearing compared with antibiotics group (flavophospholipol). Gut health could improve broiler performance, which 
balancing intestinal microflora could improve efficiency of utilization and digestibility of feed. Based on IR spectroscopy analysis, both mushroom crude extract contained $\beta$-glucan which play antioxidant and therapeutic agents in poultry diets. $\beta$-glucan contained in Agaricus bisporus and Auricularia auricula may improve broiler live weight. Dietary $\beta$ glucan could reduce digesta viscosity in small intestine, so nutrient absorption will increase with reduce organism fermentation time by endogenous enzyme (Huyghebaert et. al., 2011). Major polysaccharides in Agaricus bisporus and Auricularia auricula are $\beta$-D-glucan, which induces immune response to stimulate macrophage proliferation.

Carcass yield in Table 2 showed that Agaricus bisporus and Auricularia auricula crude extract addition in average 70.6 $73.5 \%$, which is broiler carcass yield at about $70-75 \%$. In study of $3 \%$ yeast cell wall inclusion into broiler diets did not show significant differences compared with oxytetracycline group (Ahmed et al., 2015). Kavyani et al. (2012) also reported that 3\% Agaricus bisporus inclusion in broiler diet showed $73.3 \%$ carcass yield with no significant differences between treatments. Another study showed 6\% inclusion of white button mushroom simultaneous increase broiler carcass yield (Mazaheri et al., 2014). Breast meat yield in average of 29.1 - 31.0\%, there were no significant differences among the treatment, which indicates Agaricus bisporus and Auricularia auricula crude extract did not affect on broiler breast meat conformation. Meanwhile the lowest abdominal fat percentage showed in Agaricus bisporus crude extract $1.2 \%$ inclusion with $1.19 \%$ of body weight. In addition of edible mushroom crude extract contained $\beta$-gulcan could stimulate macrophages and dendritic cell which needed more energy transferred into peyer's patches to improve host imunity by increasing CD4 and TCR 1 cells (Yalcin et. al., 2014; Chae et. al., 2006), then balancing energy storages in whole body as fat. Carcass dressing in this study ranged from $70-73 \%$, whereas similar study by Ahmed et. al. (2015) reported carcass dressing percentage in the range of $70-73 \%$ without showed significant differences between treatments using Saccharomyces cerevisiae inclusion. Saccharomyces cerevisiae cell wall is rich in $\beta$-1,3-glucan which is important for modulating immune response and nutrient utilization in the small intestine (Nie et. al., 2018).

\section{CONCLUSION}

In conclusion, absorption characteristics of the glycosidic bands in $\alpha$ or $\beta$ - type of monosaccharides of A. bisporus and $A$. auricula crude methanolic extracts were identified using FT-IR spectroscopy. Meanwhile, both edible mushroom crude extracts did not change the carcass quality of broiler.

\section{REFFERENCES}

Ahmed, M. E., Abbas, T. E., Abdlhag, M. A., \& Mukhtar, D. E. (2015). Effect of dietary yeast (Saccharomyces cerevisiae) supplementation on performance, carcass characteristics and some metabolic responses of broilers. Animal and Veterinary Sciences, 3(5), 5-10. https://doi.org/ 10.11648/j.avs.s.2015030501.12

Chae, B. J., Lohakare, J. D., Moon, W. K., Lee, S. L., Park, Y. H., \& Hahn, T.-W. (2006). Effects of supplementation of $\beta$-glucan on the growth performance and immunity in broilers. Research in Veterinary Science, 80(3), 291-298. https://doi.org/10.1016/j.rvsc.2005.07 .008

El-Batal, A. I., Azab, K. H. S. H., Saada, H. N., Rezk, R. G., \& El-Tahawy, N. A. (2008). Ameliorating effects of yeast Glucan with zinc bisglycinate on histological and biochemical changes in $\gamma$-irradiated rats. International Journal of Agriculture and Biology, 10(4), 361-368. 
Feng, Y., Li, W., Wu, X., He, L., \& Ma, S. (2010). Rapid and efficient microwave-assisted sulfate modification of lentinan and its antioxidant and antiproliferative activities in vitro. Carbohydrate Polymers, 82(3), 605-612. https://doi. org/10.1016/j.carbpol.2010.05.025

Giannenas, I., Tsalie, E., Chronis, E., Mavridis, S., Tontis, D., \& Kyriazakis, I. (2011). Consumption of Agaricus bisporus mushroom affects the performance, intestinal microbiota composition and morphology, and antioxidant status of turkey poults. Animal Feed Science and Technology, 165(3-4), 218-229. https://doi.org/10. 1016/j.anifeedsci.2011.03.002

Guimarães, J. B., dos Santos, É. C., Dias, E. S., Bertechini, A. G., da Silva Ávila, C. L., \& Dias, F. S. (2014). Performance and meat quality of broiler chickens that are fed diets supplemented with Agaricus brasiliensis mushrooms. Tropical Animal Health and Production, 46(8), 1509-1514. https://doi.org/10.1007/ s11250-014-0655-9

Hahn, T.-W., Lohakare, J. D., Lee, S. L., Moon, W. K., \& Chae, B. J. (2006). Effects of supplementation of $\beta$ glucans on growth performance, nutrient digestibility, and immunity in weanling pigs. Journal of Animal Science, 84(6), 1422-1428. https:// doi.org/10.2527/2006.8461422x

Huyghebaert, G., Ducatelle, R., \& Immerseel, F. Van. (2011). An update on alternatives to antimicrobial growth promoters for broilers. The Veterinary Journal, 187(2), 182-188. https://doi. org/10.1016/j.tvj1.2010.03.003

Kavyani, A. (2012). Evaluation of dried powder of mushroom (Agaricus bisporus) as an antibiotic growth promoter substitution on performance, carcass traits and humoral immune responses in broiler chickens. Journal of Medicinal Plants Research, 6(1), 94-100. https://doi.org/10.5897/JMP

\section{R11.1168}

Kavyani, Ashkan, Shahne, A. Z., Pourreza, J., Mohammad, S., Jalali Haji-Abadi, A., Nikkhah, M., \& Landy, N. (2014). Efficiency of different levels of mushroom (Agaricus Bisporus) on intestinal morphology and microflora of broiler chickens. Journal of Farm Animal Nutrition and Physiology, 9(1), 23-30.

Kozarski, M., Klaus, A., Niksic, M., Jakovljevic, D., Helsper, J. P. F. G., \& Van Griensven, L. J. L. D. (2011). Antioxidative and immunomodulating activities of polysaccharide extracts of the medicinal mushrooms Agaricus bisporus, Agaricus brasiliensis, Ganoderma lucidum and Phellinus linteus. Food Chemistry, 129(4), 1667-1675. https://doi.org/10.1016/ j.foodchem.2011.06.029

Liu, J., Jia, L., Kan, J., \& Jin, C. (2013). In vitro and in vivo antioxidant activity of ethanolic extract of white button mushroom (Agaricus bisporus). Food and Chemical Toxicology, 51, 310316. https://doi.org/10.1016/j.fct.2012 .10 .014

Mazaheri, A., Shams, S. M., Dastar, B., \& Zerehdaran, S. (2014). Effect of different levels of mushroom waste (agaricus bisporus) with or without probiotic on growth performance, carcass characteristics, and breast meat quality in broiler chickens. Poultry Science Journal, 2(2), 124-138.

Minato, K. I., \& Abe, C. (2013). Immunomodulating Effect of Polysaccharide. In Bioactive Food as Dietary Interventions for Arthritis and Related Inflammatory Diseases (pp. 241-250). Elsevier. https://doi.org/10. 1016/B978-0-12-397156-2.00016-8

Nie, S., Cui, S. W., \& Xie, M. (2018). BetaGlucans and Their Derivatives. In Bioactive Polysaccharides (pp. 99141). Elsevier. https://doi.org/10.10 16/B978-0-12-809418-1.00003-4

Nikonenko, N. A., Buslov, D. K., Sushko, N. I., \& Zhbankov, R. G. (2000). 
Investigation of stretching vibrations of glycosidic linkages in disaccharides and polysaccarides with use of IR spectra deconvolution. Biopolymers, 57(4), 257-262. https://doi.org/10.10 02/1097-0282(2000)57:4<257::AIDBIP7>3.0.CO;2-3

Proestos, C., \& Komaitis, M. (2008). Application of microwave-assisted extraction to the fast extraction of plant phenolic compounds. LWT Food Science and Technology, 41(4), 652-659. https://doi.org/10.1016/j.lwt .2007 .04 .013

Purwanto, H., Hartati, I., \& Kurniasari, L. (2010). Pengembangan microwave assisted extractor (MAE) pada produksi minyak jahe dengan kadar zingiberene tinggi. Jurnal Momentum UNWAHAS, 6(2), 138470.

Shamsi, S., Seidavi, A., Rahati, M., \& Nieto, J. (2015). Edible mushroom powder (Agaricus bisporus) and flavophospholipol improve performance and blood parameters of broilers. Revista Colombiana de Ciencias Pecuarias, 28(4), 291-302. https://doi.org/10.17533/udea.rccp.v2 $8 \mathrm{n} 4 \mathrm{a} 01$

Wang, L., \& Weller, C. L. (2006). Recent advances in extraction of nutraceuticals from plants. Trends in Food Science \& Technology, 17(6), 300-312. https://doi.org/10.1016/j.tifs .2005 .12 .004

Yalçin, S., Yalçin, S., Eser, H., Şahin, A., Yalçin, S. S., \& Güçer, Ş. (2014). Effects of dietary yeast cell wall supplementation on performance, carcass characteristics, antibody production and histopathological changes in broilers. Kafkas Universitesi Veteriner Fakultesi Dergisi, 20(5), 757-764. https:// doi.org/10.9775/kvfd.2014.11088

Yang, B., Zhao, G., Wang, L., Liu, S., \& Tang, J. (2020). Effects of the Agaricus bisporus stem residue on performance, nutrients digestibility and antioxidant activity of laying hens and its effects on egg storage. AsianAustralasian Journal of Animal Sciences. https://doi.org/10.5713/ajas. 19.0853

Zeng, W.-C., Zhang, Z., Gao, H., Jia, L.-R., \& Chen, W.-Y. (2012). Characterization of antioxidant polysaccharides from Auricularia auricular using microwave-assisted extraction. Carbohydrate Polymers, 89(2), 694-700. https://doi.org/10.10 16/j.carbpol.2012.03.078 